\title{
Evaluation of Cairo Squares among Different Time Periods
}

\author{
Manal Tawfik \\ Shorouk Academy
}

\begin{abstract}
Squares are considered a container of the events and activities that happen in the urban environment. But the severe deterioration of Cairo squares that is accompanied. From these points became an input for this research. The research will work by choosing ten urban squares in Cairo in different time periods that represent the urban squares in Cairo. An evaluation will be held to form some solutions to decrease the pressure on the squares' users and to fulfill their needs. The research is divided into three parts. The first part is concerned with evaluating these squares. The second part is concerned with holding a comparative study between them. The third part is the recommendations.
\end{abstract}

Keywords: Squares, Traffic paths, Creativity and Formation, Function, User, Efficiency and Continuity

\section{Introduction}

Cairo's squares have been used all through history for training, gathering and showing armies. They are also used as public parks for various celebrations, feasts and events such as sports, horsemanship and shooting. In the present period, squares suffer many urban and architectural issues which drove people away from gathering there and enjoying their time. Thus, the current study focuses on the most important squares at Cairo which belong to different epochs and represent the urban and architectural history of Cairo. These squares are different with respect to the architectural and urban formation in addition to function and importance to Cairo .This way, the urban and architectural formation of Cairo I have analysed in one of my previous research thoroughly (Manal,2017)[1] to stand upon the most pressing issues Cairo suffers from nowadays, And in this paper I will evaluate this Squares in order to find comprehensive solutions and determine the best square in the achievement of each standard of urban design standards and determine the best Square in Cairo in terms of achievable for all urban design standards. It focuses on Al-Hussein Square from Fatimid Cairo, Ramsis and Talaat Harb Squares from Cairo Kdiop, squares of Al-Korba, Roxi and Al-Hegaz at Heliopolis, As-Sa'ah Square located in Nasr City, and finally Mustafa Mahmoud and Lebanon Squares at Al-Mohandessin

\section{Evaluation of Squares}

This section is devoted to the evaluation of the squares of Cairo to stand upon the most significant problems they face. The researcher used the same evaluation form that was used by Marwa Adel in her master's thesis which she used to evaluate the Umm Kulthum Square in Mansoura City (Marwa,2016)[2]. The forms used in the evaluation of the squares were analyzed using Microsoft Excel, and the results are explained as the following:

\subsection{Evaluation of Al-Hussein Square}

The analysis of Al-Hussein Square shows that it encounters various urban issues which do not allow it to perform its role effectively. The efficiency of motion paths does not exceed $46 \%$ whereas innovation and architectural composition mount to $36 \%$ due to the existence of Al-Azhar Bridge. The latter creates a handful number of issues, the most important of which are lack of visual clarity and comfort. Notwithstanding with this, the Square succeed in achieving only $54 \%$ of its functional value due to lack of human activities. Besides, the 
design is not in line with its multi-purposes: religious, commercial and traffic which result in traffic congestion. From users' perspective, the Square's efficiency reaches $63 \%$ as it lacks thermal and audible means of comfort. It does not have any public services; consequently, the user does not contribute to its maintenance, especially, when the motion paths between different means of transportation and pedestrians are tangled. However, the Square provides its users with a sense of belonging owing to Al-Hussein Mosque which attracts people to visit it and buy inexpensive goods. Users would also feel secure because of its crowdedness all day and night. The Square's continuous efficiency in performing its job reaches $30 \%$ due to poor restoration and maintenance as there are no binding laws to govern the administrative bodies and users, in addition to lack of electronic surveillance and the poor contribution of the users in maintaining the Square.

\subsection{Evaluation of Tahrir Square}

The analysis of Tahrir Square demonstrates that the efficiency of motion paths reaches $58 \%$ because users can easily access it and other neighboring squares, in addition to provideing security. The motion paths, however, are poor because the pedestrians' motion is not secured, especially, for the disabled. Innovation and architectural composition are achieved by $89 \%$ for its clear visual perception, visual comfort, compatibility between the masses and colors in addition to the interrelation between the Square and its surroundings. The memorial scale is very significant that it stresses the functionality of the Square and the important buildings overlooking it; thus, its functional efficiency reaches $89 \%$. The Square includes signposts, so it is evident that it contains human activities and services in complementarity with its function. Social interaction is not achieved because of the current security conditions; however, it suits all its users. Owing to its political and historic significance, the Square's efficiency for users mounts to 53\% because the Square gives a sense of belonging. As for security, the Square is busy all the time due to its various uses which encourages its users to visit it several times. It is important to note that the Square's scale does not match the human's due to the poor public services in addition to lack of thermal and audible means of comfort. Besides, users do not participate in its maintenance, so its efficiency is weakened. The actual efficiency achieved reaches 95\% because of restoration, continuous maintenance, binding laws for administrative bodies and users, existence of electronic surveillance in addition to the positive attitude of the users and their contribution to its preservation.

\subsection{Evaluation of Ramsis Square}

As per the analysis of Ramsis Square, the efficiency of motion paths reaches 54\% thanks to its accessibility in addition to the secure motion from and to it and the neighboring squares. It is important to mention that unsecured motion paths and lack of pedestrians' paths, especially, for the disabled have led to inefficient motion paths. Besides, innovation and composition reach $57 \%$ because of the vibrant visual perception in addition to the interrelation between the Square and its surrounding environment. The memorial scale has a functional value as it acts as a local and regional stop, and it has several significant buildings overlooking it; nonetheless, the visual discomfort and the poor harmony between masses and colors have also weakened the innovation and composition to reach 54\%. The Square includes human activities which complete its function. Absence of signposts, social interaction and suitability for all users have undermined this functional value. The Square's efficiency for users reaches $50 \%$ because its scale is not in accordance with the human scale. There are no thermal or audible means of comfort, public services or contribution of users to preserve the Square which does not provide any sense of belonging to its users for the lack of supporting elements, heavy traffic flow and pollution. However, security is achieved because it is busy all day long with various means of transportation which connect Cairo to all the governorates; this encourages many users to visit it several times. Efficiency and continuity reach $45 \%$ owing to its poor restoration and maintenance, lack of binding laws for administrative bodies and users in addition to insufficient participation of the users in maintenance, but the electronic surveillance of the Square and the good manners of the users' behavior guarantee the continuity of its efficiency. 


\subsection{Evaluation of Talaat Harb Square}

The easy access to Talaat Harb Square and security in moving to and from it make its efficiency reach $63 \%$. Nonetheless, the Square has no secured motion path for disabled pedestrians which resulted in poor motion paths. Innovation and composition reach $82 \%$ as the visual perception is clear and comforting; besides, the harmony between masses and colors, the interrelation between the Square and its surrounding environment and the richness in architectural composition for buildings have also contributed to its innovation and composition. The importance of the memorial scale lies in its functional significance, so the functional efficiency reaches $61 \%$ due to the signposts, human activities and complementarity of the functions it was established for. Social interaction is achieved through coffee shops and restaurants, but the Square has no supplements which is considered a weakness. Furthermore, the Square's efficiency for users reaches $63 \%$ that gives a sense of belonging, especially, with its historic background, beautiful architectural composition and cleanliness. Security is attained because various activities are being practiced all day long, thus inviting more users. Nevertheless, the Square lacks facilities, harmony between its scale and the human scale, thermal and audio comfort in addition to public services. Efficiency and continuity, thus, reach $85 \%$ because of restoration acts, continuous maintenance, binding laws for administrative bodies and users in addition to the positive attitude of the users and their contributions.

\subsection{Evaluation of Al-Korba Square}

The efficiency of the Square's motion paths reaches 63\% owing to secured movement to and from it; however, lack of pedestrians' motion path, especially, for the disabled result in poor efficiency of motion. The efficiency of innovation and composition reach $89 \%$ due to clarity in visual perception and comfort, coherence between masses and colors, the interrelation between the Square and its environmental surroundings, richness in architectural composition for those buildings overlooking the Square and the existence of the human scale. The functional efficiency reaches $64 \%$ as it has signposts, coffee shops and restaurants which create social interaction. Although lack of human activities and facilities in addition to traffic congestion do not make users feel comfortable, user efficiency reaches $56 \%$ owing to its history and the beauty of its architectural composition which create a sense of belonging and security. Since the Square is crowded all the time with various activities, users are interested in visiting it, especially, that it provides the thermal comfort by using protectors from the sun. The traffic congestion hinders audible comfort, however. Generally, the Square's poor facilities do not help in offering public services, thus leading to poor efficiency. Consequently, efficiency and continuity reach $50 \%$ because of lack of constant maintenance, binding laws for administrative bodies and users in addition to lack of human's contribution to keep the place safe. It is, however, important to note that users' positive attitude and ethics contributed in raising the efficiency of the Square.

\subsection{Roxi Square}

The Square's motion paths efficiency reaches 67\%. This results from secured movement to and from the Square; however, it lacks pedestrian motion path, especially, for the disabled which lead to poor efficiency of motion. Innovation and composition reach $61 \%$ for its clear visuals and the harmony dominating the surroundings. The memorial scale is significant because the Square acts as a knot connecting several locations, especially, with the existence of plenty of stores. Nonetheless, poor visual comfort, poor harmony between masses and color in addition to the average architectural details where the compositional elements of facades are unclear and monotonous have negatively affected this aspect. Besides, the functional efficiency reaches $61 \%$ as some human activities serve some of the Square's functional purposes, but the poor facilities do not help it attain social interaction. Accordingly, the Square does not meet all the ends of the users which weakens its functional efficiency to reach 56\%. What compensates the functional efficiency is the sense of security it provides the users with because of its business. The Square's user efficiency is affected by the absence of harmony between the Square's scale and the human's, lack of thermal and audible comfort, poor public services and meagre contribution of users to its maintenance. This is further reinforced by lack of facilities, traffic congestion and pollution. It is also found that efficiency and continuity reach $40 \%$ owing to the absence of restoration and 
maintenance, binding laws for administrative bodies and users together with lack of human contribution to keep the place sound and absence of electronic surveillance.

\subsection{Al-Hegaz Square}

The evaluation of the Square demonstrates that motion paths efficiency reaches $58 \%$ because users can easily access it; besides, there are sidewalks for pedestrians. In contrast, the unsecured motion path for users and the disabled has weakened the parameter of motion path. Innovation and composition reach $64 \%$ because the Square is harmonious with the surroundings environment; also, there is coherence between the masses and the colors. However, the visual realization is poor because it mimics the architectural style of the 1970s in Egypt which makes the Square similar to others built during this era. The importance of the memorial scale lies in its functional role as a knot Square overlooking several stores. Poor architectural details and lack of diversity in architectural composition do not offer visual comfort which result in visual discomfort and lack of innovation. Its ability to host human activities reaches $61 \%$, especially, with the existence of signposts. Nonetheless, the poor facilities do not allow human and social interaction, so all its functions are not fully realized and do not suit all users. Thus, the Square's efficiency for users reaches $59 \%$ because its busy nature results in security. The Square's scale, nonetheless, is not coherent with human scale as there is thermal and audible discomfort, absence of public services and poor involvement of users in its maintenance. Furthermore, it does not stress any sense of belonging because of lack of facilities, heavy flow of traffic and pollution which have also affected its efficiency for its users. With reference to efficiency and continuity, the absence of restoration and maintenance, binding laws for administrative bodies and users, human participation in preserving the Square and electronic surveillance make it reach 50\%. Finally, users' positive outlook and ethics have always contributed in raising the efficiency of the Square.

\subsection{Evaluation of Al-Sa'ah Square}

Motion paths' efficiency reaches $63 \%$ due to its accessibility, the availability of sidewalks for pedestrians; its only problem is that there are no secure path for pedestrians, particularly, the disabled which affects the motion path parameter. As for innovation and composition which reach $43 \%$, it is obvious that it is poor because of the visual discomfort, especially, that it follows the architectural style which dominated Egypt in the 1970s. The memorial scale gives the Square its functional value as a knot with many stores; however, poor architectural details and diverse compositional structure lead to visual uneasiness. Though the latter is accentuated by lack of coherence between masses and colors, the Square's harmony with its surroundings have compensated this aspect. The realization of the functional efficiency is $56 \%$ due to traffic jam and the incomplete performance of its functions. The Square also has poor social interaction and does not support all its users; however, the clear signposts have increased its functional efficiency to users which reaches $44 \%$. This result is due to lack of harmony between its scale and the human scale, lack of thermal and audible comfort, poor public services and poor involvement of users in its maintenance. The belonging scale is weak as well, as a result of the poor facilities, heavy flow of traffic and pollution. Being a busy Square, it is safe which makes it more convenient to its users. Efficiency and continuity reach $35 \%$ because there is no restoration or maintenance; besides, there are no binding laws for administrative bodies and users who do not participate in preserving the Square and the absence of electronic surveillance.

\subsection{Mostafa Mahmoud Square}

The analysis shows that the efficiency of motion paths reaches $67 \%$ which is a result of its accessibility, security and sidewalks; for this aspect, the drawback is the absence of pedestrian motion path, particularly, the disabled which negatively affects it. Innovation and composition reach $39 \%$ as there is no definite architectural composition because the facades overlooking the Square are similar in height and composition, or clear visual perception. This is also due to the mismatch between the masses and the colors. As the Square is considered a knot connecting several stores and locations, visual discomfort prevails which have negatively affected that aspect; however, the Square is coherent with the surrounding environment. Its success in achieving its functions 
reaches $75 \%$ due to the existence of a landmark such as Moustafa Mahmoud Mosque which contributes to the existence of human activities. The latter facilitates social interaction and promotes diversity of usage all day long; it also leads to complementarity between design and function. Notwithstanding with this, the poor facilities and unsuitability for all users do not allow the full recognition of the Square's efficiency in terms of function. As for the efficiency for users, it is achieved by $66 \%$ which recognizes belonging, fostered by Mostafa Mahmoud Mosque that offers health, social and humanitarian services; it provides as well a sense of security because it offers support equally to all the Square's users all day long. However, the inappropriateness of the Square's scale with the human values, the absence of thermal and audible comfort, lack of users' contribution to the preservation of the Square, the nonexistence of facilities, traffic jam and pollution have weakened the Square's efficiency. The parameter of efficiency and continuity, thus, reaches $40 \%$ due to the absence of restoration or maintenance, binding laws for administrative bodies and users who do not take good care of the Square and electronic surveillance.

\subsection{Lebanon Square}

Motion paths' efficiency reaches $54 \%$ as the Square is within the reach of the users, particularly, for being security and having sidewalks; however, it has no special motion path for the disabled which fails this aspect. As the visual aspect is undermined because the architectural style is similar to that of the 1970s, the efficiency of innovation and composition mounts to 39\%, specially, that the Square is similar to all the 1970s squares. It derives its memorial scale from functioning as a knot Square where many stores overlook it, creating visual discomfort; the latter results from free-of-architectural details facades that lack diversity in its composition and harmony between masses and colors which have contributed to the poor innovation and composition. The functional efficiency scores $43 \%$ because it does not contain human activities or shows full complementarity with its functions; it also has no signposts. Lack of facilities does not result in social interaction and weakens its usefulness to users. The efficiency for users reaches $38 \%$ because the Square's scale does not match the human scale. The Square, in this respect, does not provide thermal and audible comfort, public services; the users do not even contribute to its preservation. There is, hence, no sense of belonging, specially, with lack of facilities; in addition, traffic jam and pollution have fostered this. As the Square is totally busy, its efficiency to users increases.

\section{A Comparative Study for Squares' Efficiency in Achieving Each Design Standard:}

The forms used in the evaluation of the squares were analyzed using Microsoft Excel, and the results are explained in the chart below:

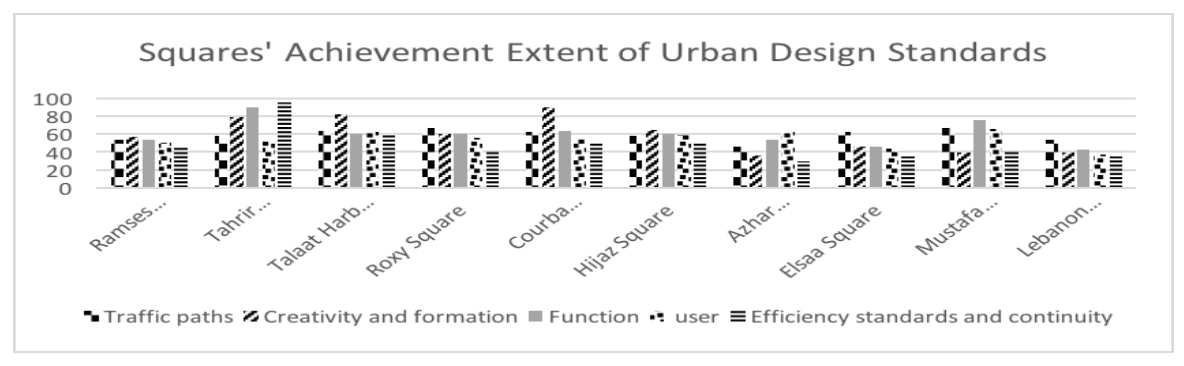

Fig. 1: A Comparative Study for Squares' Efficiency in Achieving Each Design Standard

The comparison between the extent to which urban design standards are achieved and urban spaces shows in the Fig 1 that the best squares in terms of motion paths are Roxi and Al-Hegaz as their evaluation criteria is $67 \%$. The best square in creation and formation is Al-Korba followed by Talaat Harb as the percentage is $89 \%$ for AlKorba Square and $82 \%$ for Talaat Harb Square. As for the standard of function performance, the best square is Tahrir Square which achieved 89\%; besides, Al-Azhar Square and Tahrir Square are the best in terms of users as it reaches $63 \%$. Tahrir Square is considered the best square with respect to efficiency and continuity. 


\section{A Comparative Study of Squares' Efficiency in Achieving the Standard of Design}

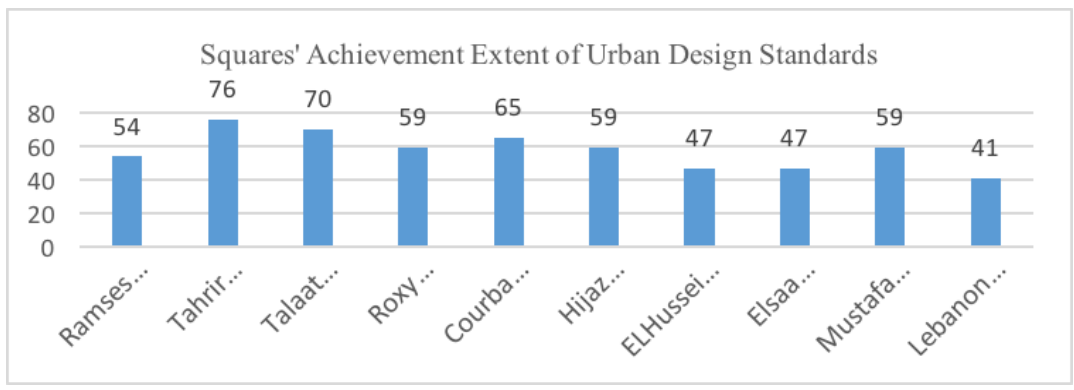

Fig. 2: A Comparative Study of Squares' Efficiency in Achieving the Standard of Design

The comparative study shows in Fig 2 that Tahrir Square is the best in this respect as its success reaches 76\%. In the second place is Talaat Harb Square with $70 \%$ followed by Roxi Square, Al-Hegaz Square and Mostafa Mahmoud Square for 59\%, Ramsis Square for 54\%. Finally, the Squares of Al-Hussein, Al-Sa'ah and Lebanon are the least efficient in terms of achieving the standards of urban design for squares.

\section{Conclusions}

The squares of Cairo were established in different epochs, and they encounter various urban issues which can be summed as follows:

\subsection{Movement Paths:}

- Complexity of traffic flow due to lack of organization for the movment paths whether for cars or public transportation.

- Absence of pedestrian paths except for a few pavements used by street vendors; this leads to confusion in the movment of cars with pedestrians which result in the slow flow of traffic.

- Absence of bicycling lanes and paths in order to reduce the usage of bicycles despite its importance in solving the problems of Cairene streets and squares through the reduction of reliance on public transportation and cars.

- Taxi and public transportation drivers do not stick to drop off the passengers at the stops which causes traffic jams.

- Second row parking for cars which leads to traffic jam at the branching off streets and squares.

\subsection{Architectural Formation:}

The surrounding urban environment for the squares are distorted for the following reasons:

- The fact that there are no binding laws to determine the urban formation for the walls of squares causes absence of unified definite style for the squares which were created in the fifties of the last century, further leading to a distortion of the visual image.

- Squares established during the Kdiop Cairo period does not suffer this issue because its design is already definite and distinguished from the start. In addition, the government pays much attention to this area, makes projects to revive it and puts laws to protect the area from visual and architectural distortion.

- Al-Korba Square is branded for its distinguished architectural formation, but the lack of binding laws and the country's carelessness about protecting it as is the case with Kdiop Cairo resulted in visual impairment for the Square and the whole area since the establishment of extra floors and nests at the top of the historic sites.

- The absence of binding laws in the design of display windows and banners for stores, coffee shops and restaurants which caused distortions in the visual image.

- Squares established since the 70 s have poor facades in terms of beauty as they lack distinctive architectural styles. 


\subsection{Function}

- Squares at Cairo have various functions such as commercial, administrative, touristic and traffic. The squares have succeeded in fulfilling most of their functions like administrative, trade and touristic functions meanwhile they suffer traffic issues because uses overlap with traffic flow.

- The furnishing of the squares is very poor because seats for pedestrians barely exist.

- Most of the squares have attained the parameter of social interaction through the existence of restaurants, coffee shops and stores which encourage socialization.

\subsection{The User}

- Thermal and acoustic comfort are not provided at the squares due to heavy traffic flow and lack of public services which diminishes the belonging factor; nonetheless, they are characterized by security because the functions of squares entail mobility all day long.

\subsection{Efficiency and Continuity:}

- No maintenance or restoration activities are taking place at these squares except for Kdioip Cairo revival project which focuses on Kdioip Cairo squares.

- There are no binding laws for the administrative bodies and the user in order to preserve the urban environment.

- Users do not contribute to the preservation of the squares due to lack of public services or furnishing which enable the users to sit and relax. Also, the disturbances and the pollution do not encourage users to preserve it.

\section{Recommendations}

1. Separating movement paths should be separated as there should be special paths for public transportation, cars, bicycles and pedestrians.

2. Paying greater attention to providing squares with seats and lighting columns to encourage pedestrians to have picnics and visit the squares.

3. Giving more attention to green lands, trees and coordination since they represent the cultural image for the city.

4. Improving and developing the condition of the pavements and the streets looking over it.

5. Providing public services for the users such as public bathrooms so that they do not defecate in the street which increases pollution.

6. Preventing stores, coffee shops and street vendors from infringing upon the pedestrians' pavements to put an end to the distortions of environment and users' reluctance to walk on pavements which causes traffic congestion when they walk among the cars.

7. Providing electronic surveillance to protect the squares from abuse whether by passers-by or drivers.

8. Putting binding laws for users and administrative bodies.

9. Giving attention to the restoration and maintenance to guarantee efficiency and continuity.

10. Setting legislations and laws with respect to the fundamentals of architectural formation for buildings and stores overlooking the squares to protect the architectural style from distortion.

11. Setting rules for the use of horns to maintain quietness.

\section{References}

[1] M,Tawfik, Observation, analysis and evaluation of Cairo squares among different time periods,2017.

[2] M, Adel, Sustainability of Forming Thought for Heritage Urban, Spaces ,An analytic Study for Heritage squares ,Master, Mansoura University, p100-101, 2016.

[3] Sit Visit,2016 